\title{
$X$-ray Computed Tomography Instrument Performance Evaluation, Part II: Sensitivity to Rotation Stage Errors
}

\author{
Bala Muralikrishnan, Meghan Shilling, Steve Phillips ${ }^{1}$, Wei Ren, Vincent Lee, and Felix Kim \\ National Institute of Standards and Technology, \\ Gaithersburg, MD 20899 USA \\ balam@nist.gov \\ katharine.shilling@nist.gov \\ wei.ren@nist.gov \\ vincent.d.lee@nist.gov \\ felix.kim@nist.gov

\begin{abstract}
The development of standards for evaluating the performance of X-ray computed tomography (XCT) instruments is ongoing within the American Society of Mechanical Engineers (ASME) and the International Organization for Standardization (ISO) working committees. A key challenge in developing documentary standards is to identify test procedures that are sensitive to known error sources. In Part I of this work, we described the effect of geometry errors associated with the detector and determined their influence through simulations on sphere center-to-center distance errors and sphere form errors for spheres located in the tomographically reconstructed measurement volume. We also introduced a new simulation method, the single-point ray tracing method, to efficiently perform the distance and form error computations and presented data validating the method. In this second part, also based on simulation studies, we describe the effect of errors associated with the rotation stage on sphere center-to-center distance errors and sphere form errors for spheres located in the tomographically reconstructed measurement volume. We recommend optimal sphere center locations that are most sensitive to rotation stage errors for consideration by documentary standards committees in the development of test procedures for performance evaluation.
\end{abstract}

Key words: cone-beam; distance error; documentary standards; form error; geometry errors; performance evaluation; radiographbased method; sensitivity analysis; single-point ray tracing method; X-ray computed tomography.

Accepted: April 24, 2019

Published: July 11, 2019

https://doi.org/10.6028/jres. 124.015

\section{Introduction}

A key aspect to developing documentary standards for performance evaluation is the design of test procedures that are sensitive to known error sources. Currently, standards groups within the American Society of Mechanical Engineers (ASME) and the International Organization for Standardization (ISO) are discussing test procedures to include in documentary standards for performance evaluation of X-ray computed tomography (XCT) instruments. In Part I [1] of this two-part study, we recommended optimal placement of spheres in the measurement volume so that sphere center-to-center distance errors and sphere form errors are most sensitive to geometry errors associated with the detector. We presented a novel method, called the single-point ray tracing method, that efficiently estimates these sensitivities. In this second part, we discuss optimal placement of spheres in the measurement volume so that the sphere centerto-center distance errors and sphere form errors are most sensitive to errors associated with the rotation

${ }^{1}$ Deceased. 
stage. We make recommendations on sphere center locations for consideration by documentary standards committees that are involved in the development of performance evaluation test procedures for XCT instruments. For our purposes in this paper, form error is considered to be the difference between the maximum and minimum residuals from a least-squares best-fit sphere fit to given point cloud data associated with a spherical surface.

We refer to Part I [1] for the coordinate system and the reference object used, and for a description of the single-point ray tracing method. We refer to Marsh [2] for an overview of rotation stage error sources. More specific to XCT instruments, Ferrucci et al. [3] have addressed the topic of sphere center-to-center distance errors, radius errors, and form errors in the presence of rotation stage errors by considering a reference object composed of 27 spheres arranged in a helical pattern. The objective of their research was to determine the highest magnification position for which the modelled rotation stage errors had negligible effect on dimensional measurements. For that purpose, their simulations considered all rotation stage errors together, not in isolation. Thus, they did not provide recommendations on sphere center locations that are most sensitive to individual rotation stage error sources. However, we do acknowledge that the publications by Ferrucci et al. [3, 4] serve as the basis for work reported here and in Part I [1], and that our parameterization of XCT rotation stage errors is also based on Ferrucci et al. [3].

This paper is organized as follows. We discuss errors associated with the rotation stage in Sec. 2. Discussion and conclusions follow in Sec. 3 and Sec. 4, respectively.

\section{Error Sources Associated with the Rotation Stage}

The location of the sample stage rotation axis is a quantity required during the reconstruction process. This is typically determined by a geometrical calibration process. In this section, we quantify the effects of error in the rotation axis position and in the error motions of the rotation stage (i.e., axial, radial, wobble, and encoder scale $[2,3]$ ) on sphere center-to-center distance errors and sphere form errors. Figure 1 depicts the different rotation stage error sources considered in this paper.

We note that the definition of the coordinate system given in Part I defines the rotation stage axis as orthogonal to the $Z$ and $X$ axes, and hence any fixed tilt error with respect to the detector appears as a detector angular error and not as a rotation stage error. The sensitivity values reported in this section are for the XCT instrument rotation axis and detector distances from the X-ray source focal spot of $400 \mathrm{~mm}$ and $1177 \mathrm{~mm}$, respectively. The sign convention for the errors is as described in Part I, Sec. 3.

As in the case of detector error sources, the magnitude of the simulated geometry errors is intentionally large and was determined by trial and error to provide easily observable sphere center-to-center distance errors or sphere form errors. Sensitivity is reported as $\mathrm{mm} / \mathrm{mm}$ and $\mathrm{mm} /{ }^{\circ}$ depending on the units of the geometry error source, i.e., linear or angular. Such a sensitivity statement implies that the dimensional errors are linearly proportional to the magnitude of the geometry error sources. This linearity was confirmed by simulating various error source magnitudes and observing the resulting dimensional errors.

\subsection{Rotation Stage $Z$ Location Error}

Description: This parameter describes an error in the location of the measurement volume center $P$ (which is the intersection of the rotation axis and the line from the source to the detector along the $Z$ axis); see Fig. 1(a).

Distance error: This error is negatively correlated with the detector $Z$ location error (see Part I), and the ratio of the sensitivity coefficients for the two cases is equal to the magnification factor. The negative sign of the correlation arises because moving the rotation axis closer to the source has the same effect as moving the detector away from the source; i.e., both result in an increase of the magnification factor. Figure 7(a) in Part I [1] showed the lines that are most sensitive to this error source. These are diagonal lines joining spheres farthest apart from each other and passing through the center of the measurement volume $P$. 


\section{Journal of Research of the National Institute of Standards and Technology}

We use the term body diagonal to refer to these lines. Two such body diagonals are shown in Figs. 2(a) and (b). The distance error for these lines is approximately $-0.017 \mathrm{~mm}$ for $-0.1 \mathrm{~mm}$ error in the rotation axis $Z$ location and $+0.017 \mathrm{~mm}$ for $+0.1 \mathrm{~mm}$ error in the rotation axis $Z$ location. The magnitude of the distance error sensitivity is thus $0.17 \mathrm{~mm} / \mathrm{mm}$. This sensitivity is larger than the sensitivity for detector $Z$ location error (see Sec. 3.3, Part I) by a factor approximately equal to the magnification value of 2.94 .

Form error: This error source does not result in any noticeable form error in the spheres.

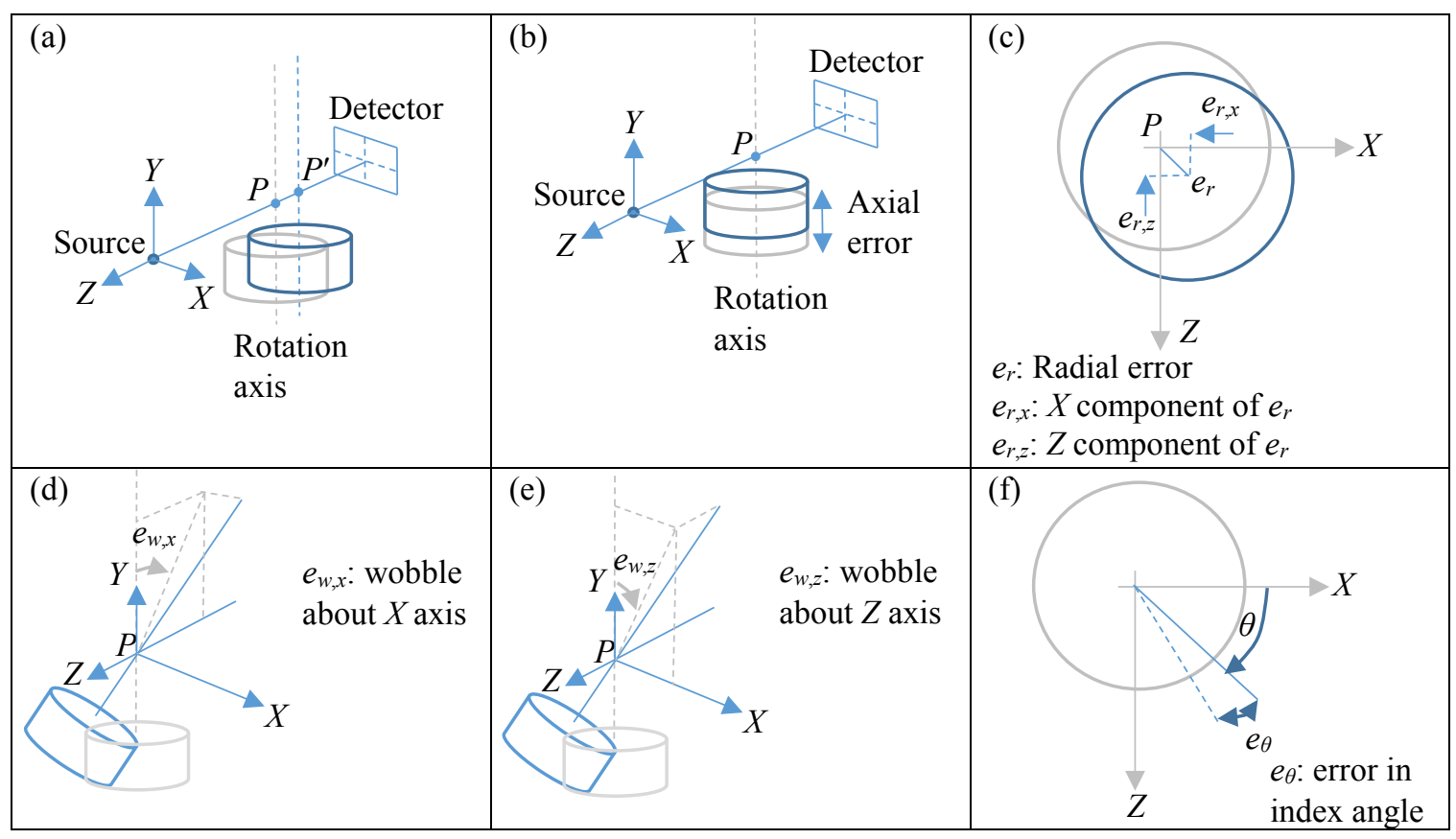

Fig. 1. Rotation stage errors. (a) Error in the location of the rotation axis along $Z$. The location of point $P^{\prime}$ along the $Z$ axis is used in the reconstruction, whereas $P$ is the true location of that point. (b) Axial error motion of the rotation stage, i.e., errors along the $Y$ direction at a given index angle $\theta$. (c) Radial error motion of the rotation axis at a given index angle $\theta$; this error can be parameterized as two separate components along $X$ and $Z$. (d) The $X$ component of the wobble error of the rotation stage (i.e., component of axis tilt in the $Y Z$ plane) for a given index angle $\theta$. (e) The $Z$ component of the wobble error of the rotation stage (i.e., component of axis tilt in the $X Y$ plane) for a given index angle $\theta$. (f) Error in the angular position of the rotation stage for a given index angle $\theta$.

\subsection{Axial Errors}

Description: Axial error is the error motion of the rotation stage along the $Y$ direction, i.e., parallel to the axis of rotation; see Fig. 1(b). The magnitude of the axial error is a function of the rotation stage index angle $\theta$. We consider individual harmonic components of the axial error of the form $a \cos n \theta$ and $a \sin n \theta$, where $a$ is the amplitude, $n$ is the order of the harmonic, and $\theta$ is the index angle. Note that $\theta=0^{\circ}$ is along the positive $X$ axis. The amplitude $a$ is $0.1 \mathrm{~mm}$, and we consider low-order harmonics $n$ ranging from 1 to 10.

Distance error: Figures 2(a) and (b) show the lines of maximum sensitivity for the first-order axial error motion case; these are the body diagonals passing through $P$. In the case of the cosine term, the lines are located on the $Y Z$ plane, whereas in the case of the sine term, the lines are located on the $X Y$ plane. The magnitude of the distance error is $0.01 \mathrm{~mm}$; the distance error sensitivity is therefore $0.1 \mathrm{~mm} / \mathrm{mm}$. The error is positive for one of the lines and negative for the other. The sign of the distance error reverses when the amplitude of the axial error is $-0.1 \mathrm{~mm}$. Higher-order harmonics did not result in a distance error.

Form error: Because axial errors result in all spheres moving up and down along the $Y$ axis, the resulting spheres appear elongated along the $Y$ direction, with the magnitude, number, and orientation of 
the lobes depending on the order of the harmonic. The magnitude of the form error is approximately 0.2 $\mathrm{mm}, 0.16 \mathrm{~mm}, 0.14 \mathrm{~mm}, 0.12 \mathrm{~mm}$, and $0.1 \mathrm{~mm}$ for the second-, fourth-, sixth-, eighth-, and tenth-order (both cosine and sine) terms, respectively. The form error is approximately $0.1 \mathrm{~mm}$ for all odd-order terms. The largest sensitivity is therefore approximately $2 \mathrm{~mm} / \mathrm{mm}$ for the cases of the second-order sine and cosine terms. For any given order, the form error is identical for all spheres. Figure 3 shows the form error for the second- and fourth-order cosine and sine terms.

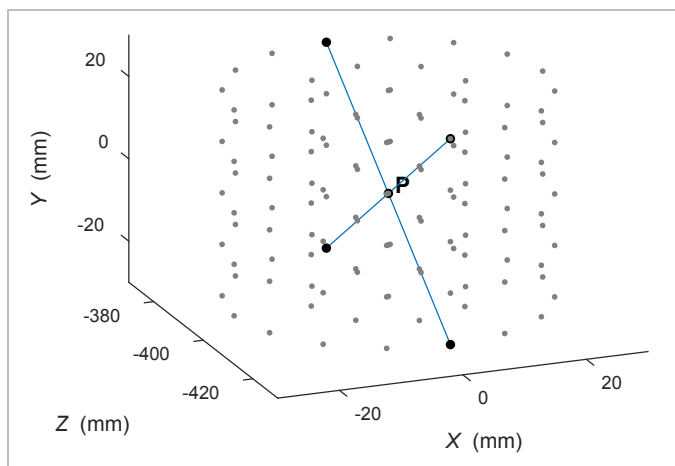

(a)

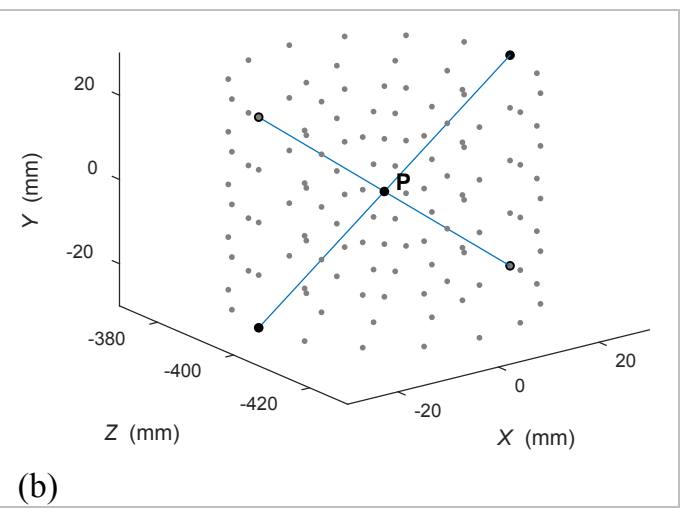

Fig. 2. The lines of maximum sensitivity for distance errors for (a) first-order cosine, and (b) first-order sine axial error motion.

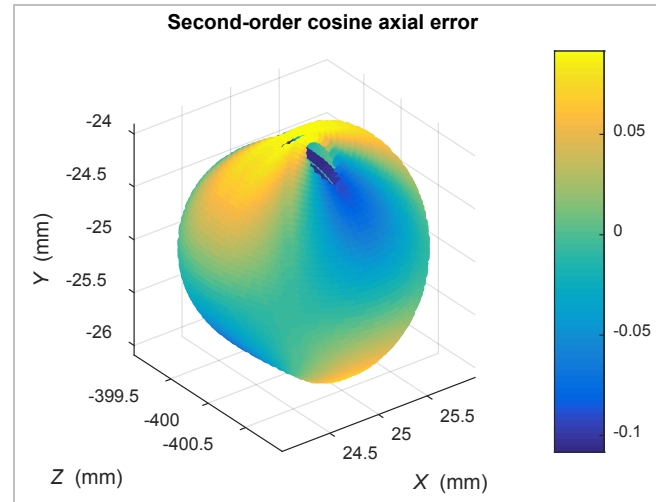

(a)

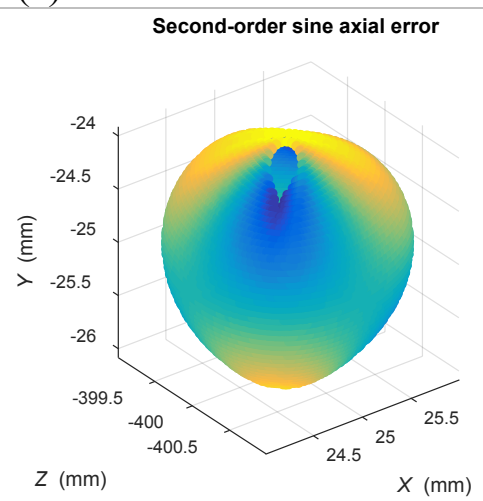

(c)

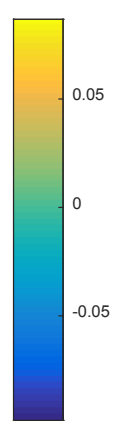

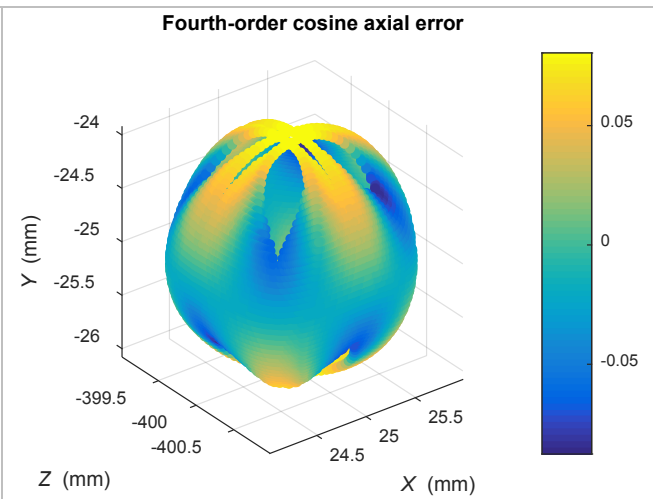

(b)

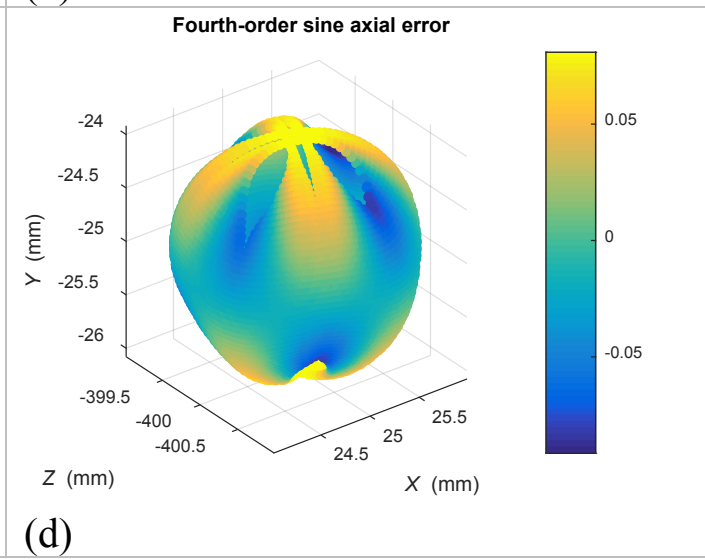

Fig. 3. Form error in the presence of (a) second-order cosine, (b) fourth-order cosine, (c) second-order sine, and (d) fourth-order sine axial error. 


\subsection{Radial Error Component Along the $X$ Axis}

Description: Radial error is the error motion of the rotation stage in the $X Z$ plane, i.e., perpendicular to the rotation axis; see Fig. 1(c). The magnitude of the radial error is a function of the rotation stage index angle $\theta$. This error motion is resolved into components along the $X$ and the $Z$ axes. We discuss the $X$ component of radial error here and the $Z$ component in the next subsection. As in the case of axial errors, we consider individual harmonic components of the $X$ component of the radial error, which have the form $a \cos n \theta$ and $a \sin n \theta$, where $a$ is the amplitude, $n$ is the order of the harmonic, and $\theta$ is the index angle. In this study, the amplitude $a$ is $0.1 \mathrm{~mm}$, and we consider low-order harmonics $n$ ranging from 1 to 10 .

Distance errors: Figure 4 shows the lines with the highest sensitivity to the second-order cosine and sine terms of the radial error motion component along $X$. These lines join spheres located diametrically apart from each other on any given horizontal $(X Z)$ plane. We use the term face diagonals to refer to such lines. The magnitude of the distance error is approximately $0.01 \mathrm{~mm}$; the distance error sensitivity is therefore $0.1 \mathrm{~mm} / \mathrm{mm}$. The sign of the distance error reverses when the amplitude of the radial error is -0.1 $\mathrm{mm}$. There are no significant distance errors for any other harmonic component.

Form error: Because radial errors result in all spheres moving radially together, all spheres appear elongated in the horizontal $(X Z)$ plane, with the magnitude, number, and orientation of the lobes depending on the order of the harmonic. The magnitude of the form error for the first-order sine and cosine terms is negligibly small. For all other orders, the form error is at least $0.1 \mathrm{~mm}$. The magnitude of the form error is approximately $0.2 \mathrm{~mm}, 0.2 \mathrm{~mm}, 0.16 \mathrm{~mm}$, and $0.14 \mathrm{~mm}$ for the third-, fifth-, seventh-, and ninth-order (both cosine and sine) radial error motion components along the $X$ axis, respectively. The form error is approximately $0.1 \mathrm{~mm}$ for all even-order terms. The largest sensitivity is therefore about $2 \mathrm{~mm} / \mathrm{mm}$ for the case of the third- and fifth-order sine and cosine terms. For any given order, the form error is identical for all spheres. Figure 5 shows the form error for the third- and fifth-order cosine and sine radial error motion components along the $X$ axis.

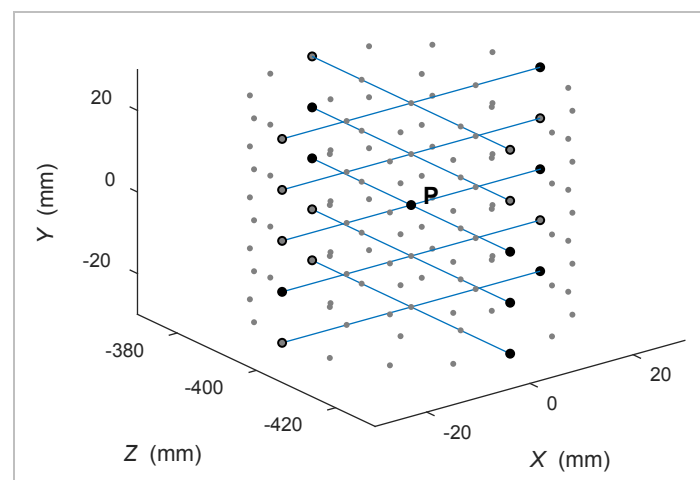

(a)

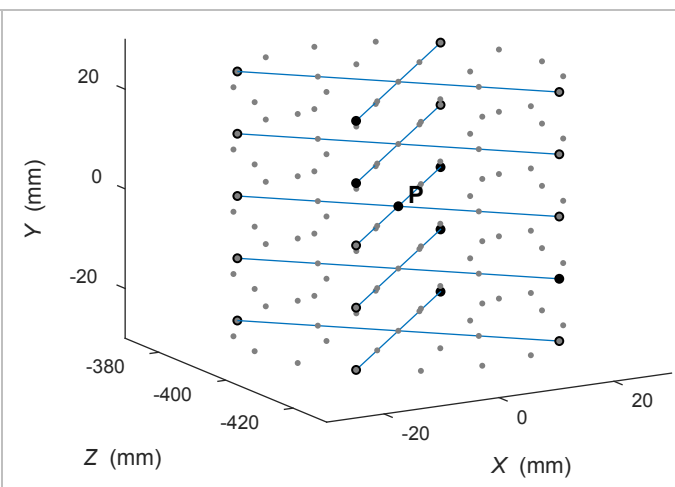

(b)

Fig. 4. Lines of highest sensitivity for (a) second-order sine, and (b) second-order cosine radial error motion along $X$.

\subsection{Radial Error Component Along the $Z$ Axis}

Description: We discuss the $Z$ component of radial error here, see Fig. 1(c). As in the previous case, we consider individual harmonic components of the $Z$ component of the radial error, which is of the form $a \cos n \theta$ and $a \sin n \theta$, where $a$ is the amplitude, $n$ is the order of the harmonic, and $\theta$ is the index angle. In this study, the amplitude $a$ is $0.1 \mathrm{~mm}$ and we consider low order harmonics $n$ ranging from 1 to 10 .

Distance errors: For the second order harmonic component, distance errors were approximately 0.01 mm with Fig. 4(a) showing the sensitive lines for the cosine term and Fig. 4(b) showing the sensitive lines for the sine term. There are no significant distance errors for any other harmonic component. 
Form error: There is no discernable form error in the spheres resulting from the radial error component along the $Z$ direction. This error source simply results in the spheres appearing larger or smaller, without significant error in the center location in the radiographic image for any index angle $\theta$.

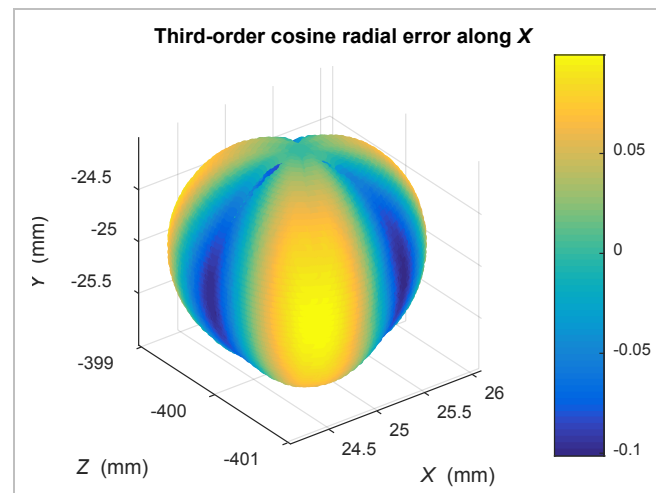

(a)

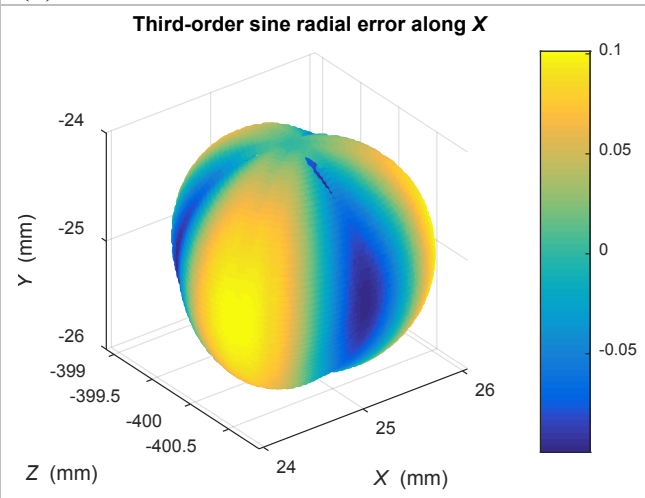

(c)

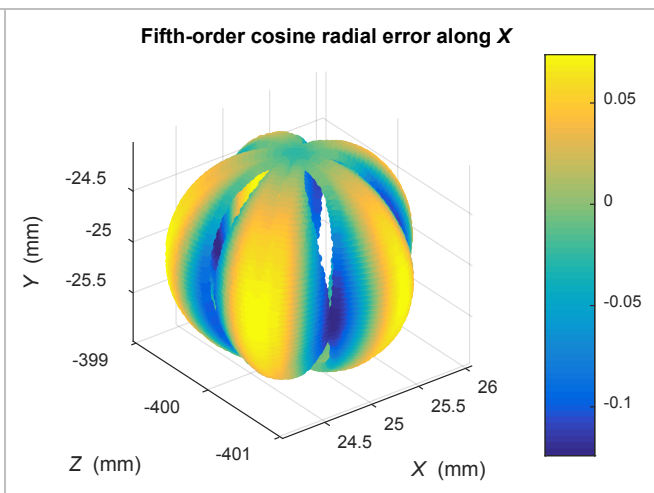

(b)

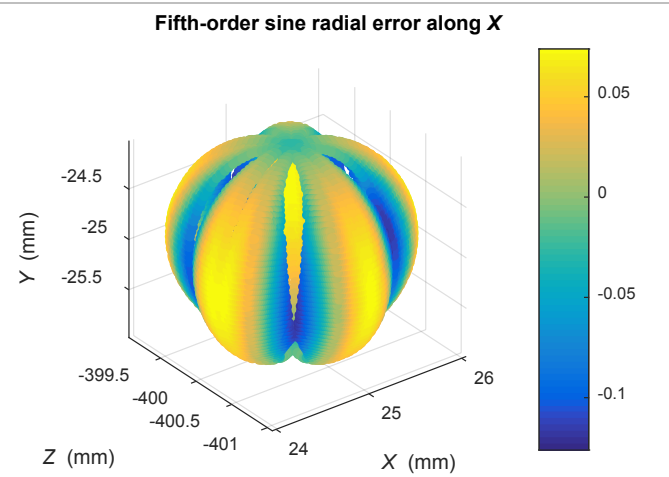

(d)

Fig. 5. Form error in the presence of (a) third-order cosine, (b) fifth-order cosine, (c) third-order sine, and (d) fifth-order sine radial error motion along the $X$ axis.

\subsection{Wobble about the $X$ Axis}

Description: Wobble is the tilt of the rotation axis as a function of index angle $\theta$. We resolve this tilt into two components - rotations about two fixed axes parallel to the global $X$ and $Z$ coordinate axes and passing through $P$. In this section, we consider wobble about the fixed axis parallel to the $X$ axis, with the pivot point located at the center $P$ of the measurement volume; see Fig. 1(d). We consider individual harmonic components of the form $a \cos n \theta$ and $a \sin n \theta$, where $a$ is the amplitude, $n$ is the order of the harmonic, and $\theta$ is the index angle. The amplitude $a$ is $0.2^{\circ}$, and we consider low-order harmonics $n$ ranging from 1 to 10 . Zero-order wobble is a constant tilt of the rotation stage as a function of azimuth and is indistinguishable from a tilted part mounted on a perfectly rotating stage [3].

Distance error: Sensitive lines for first-order cosine and sine wobble about a local $X$ axis are the body diagonals shown in Figs. 2(a) and (b), respectively. The magnitude of the distance error is $0.06 \mathrm{~mm}$; the distance error sensitivity is therefore $0.3 \mathrm{~mm} /{ }^{\circ}$. The error is positive for one of the lines and negative for the other. The sign of the distance error reverses when the amplitude of the wobble error is $-0.2^{\circ}$. Second-order harmonics result in a distance error of about $0.01 \mathrm{~mm}$, while higher-order harmonics have negligible distance errors.

Form error: The magnitude, number, and orientation of the resulting lobes depend on the order of the harmonic and the distance of the sphere from the axis of rotation. The magnitude of the maximum form 
error observed is approximately $0.1 \mathrm{~mm}, 0.15 \mathrm{~mm}, 0.14 \mathrm{~mm}, 0.12 \mathrm{~mm}$, and $0.1 \mathrm{~mm}$ for first-, third-, fifth-, seventh-, and ninth-order (both cosine and sine) wobble, respectively. The form error is about $0.1 \mathrm{~mm}$ for even-order wobble. The largest sensitivity is therefore approximately $0.75 \mathrm{~mm} /{ }^{\circ}$ for third-order sine and cosine wobble. For any given order, the form error is larger for spheres that are radially farther away from the axis of rotation. Figure 6 shows the form error for third- and fifth-order sine and cosine wobble for the sphere located at $(0,-25,-375) \mathrm{mm}$.

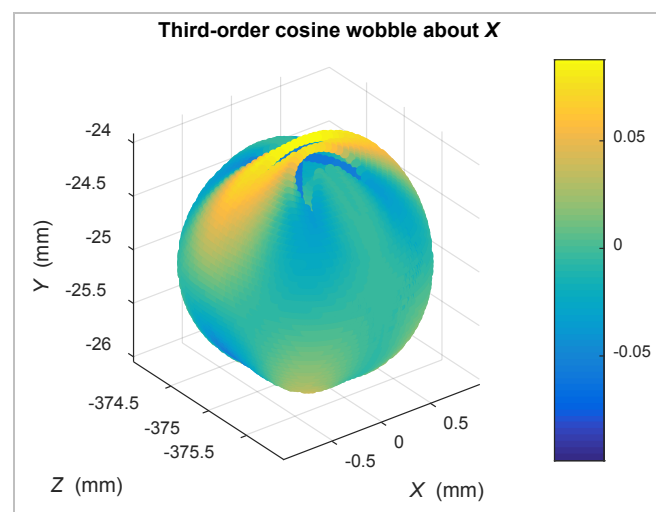

(a)

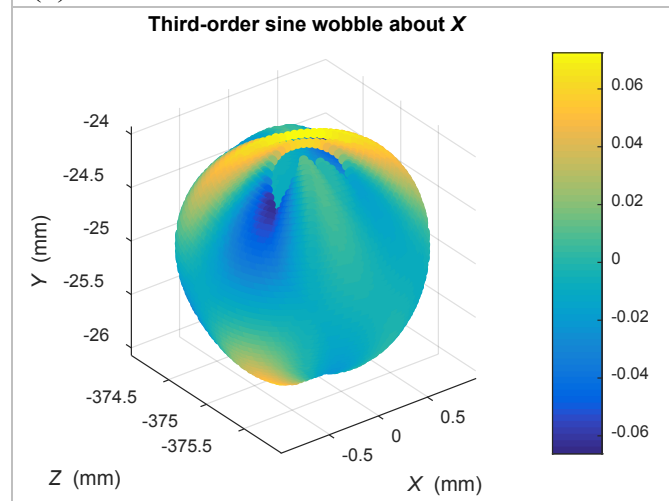

(c)

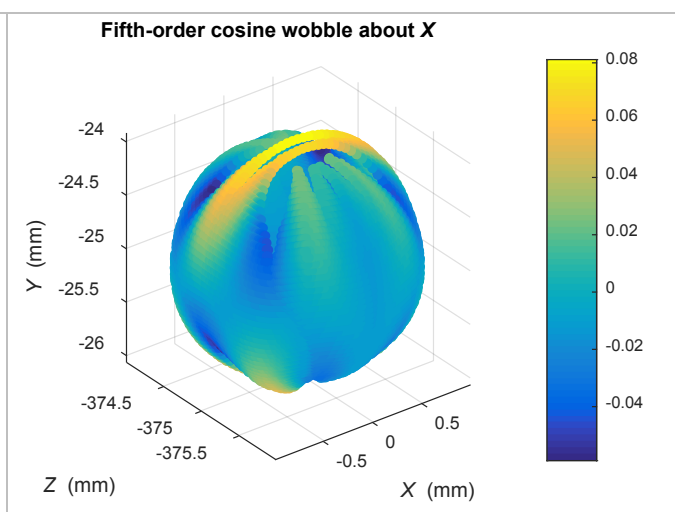

(b)

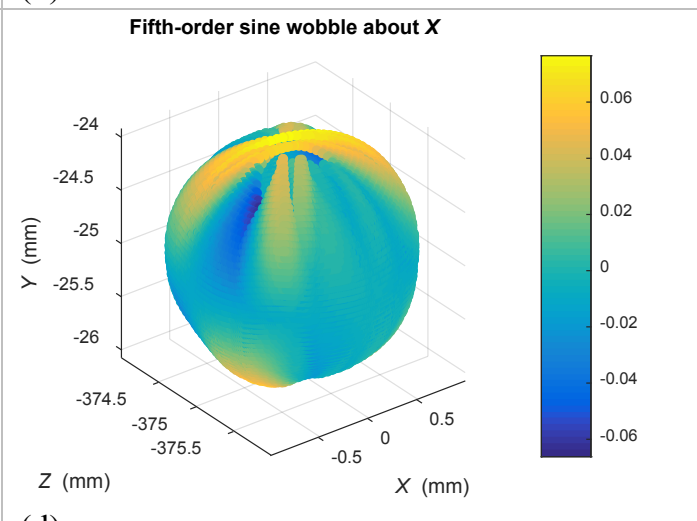

(d)

Fig. 6. Form error for the sphere located at $(0,-25,-375) \mathrm{mm}$ in the presence of (a) third-order cosine, (b) fifth-order cosine, (c) third-order sine, and (d) fifth-order sine wobble about the $X$ axis.

\subsection{Wobble about the $Z$ Axis}

Description: In this section, we consider wobble about the fixed axis parallel to the global $Z$ axis and passing through $P$; see Fig. 1(e). We consider individual harmonic components of the form $a \cos n \theta$ and $a \sin n \theta$, where $a$ is the amplitude, $n$ is the order of the harmonic, and $\theta$ is the index angle. The amplitude $a$ is $0.2^{\circ}$, and we consider low-order harmonics $n$ ranging from 1 to 10 . Zero-order wobble is a constant tilt of the axis as a function of azimuth and is indistinguishable from a tilted part mounted on a perfectly rotating stage.

Distance error: Sensitive lines for first-order cosine and sine wobble about a local $Z$ axis are the body diagonals shown in Figs. 2(b) and (a), respectively. The magnitude of the distance error is $0.06 \mathrm{~mm}$; the distance error sensitivity is therefore $0.3 \mathrm{~mm} /{ }^{\circ}$. The error is positive for one of the lines and negative for the other. The sign of the distance error reverses when the amplitude of the wobble error is $-0.2^{\circ}$. Higher-order harmonics result in negligible distance error. 
Form error: The magnitude, number, and orientation of the resulting lobes depend on the order of the harmonic and the distance of the sphere from the axis of rotation. The magnitude of the form error is approximately $0.1 \mathrm{~mm}, 0.24 \mathrm{~mm}, 0.2 \mathrm{~mm}, 0.19 \mathrm{~mm}$, and $0.17 \mathrm{~mm}$ for first-, third-, fifth-, seventh-, and ninth-order (both cosine and sine) wobble, respectively. The form error is about $0.13 \mathrm{~mm}$ for an even-order wobble. The largest sensitivity is therefore approximately $1.2 \mathrm{~mm} /{ }^{\circ}$ for the third-order sine and cosine wobble. For any given order, the form error is larger for spheres that are radially farther away from the axis of rotation. Figure 7 shows the form error for the third- and fifth-order sine and cosine wobble for the sphere located at $(0,-25,-375) \mathrm{mm}$.

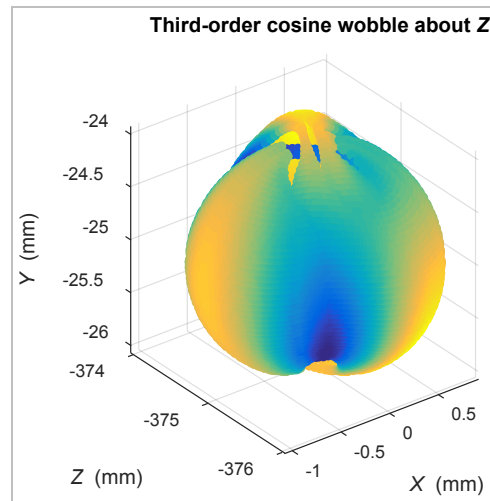

(a)

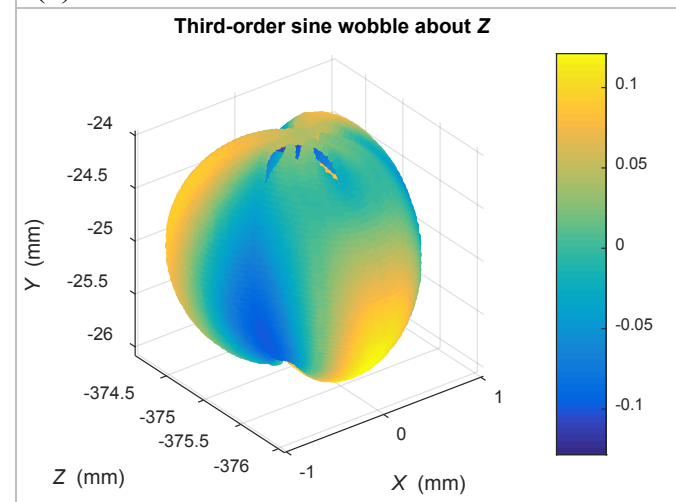

(c)

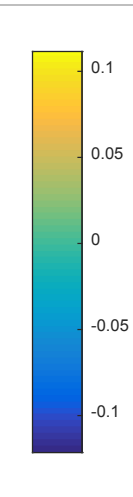

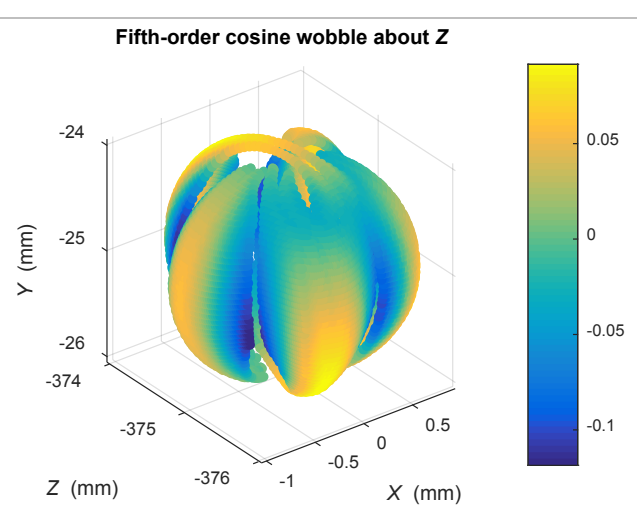

(b)

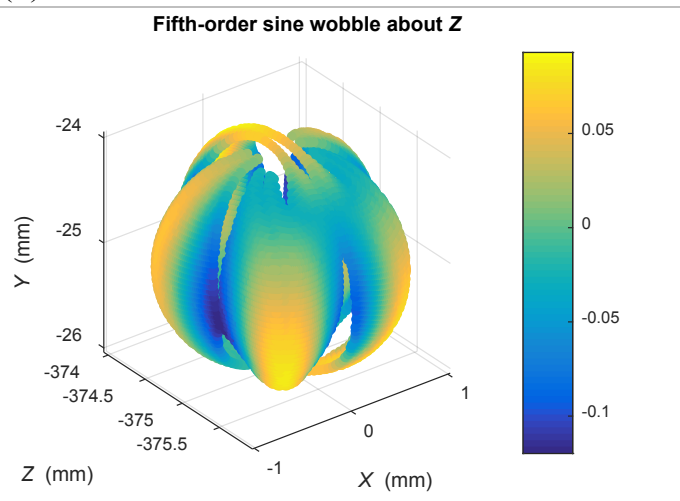

(d)

Fig. 7. Form error for the sphere located at $(0,-25,-375) \mathrm{mm}$ in the presence of (a) third-order cosine, (b) fifth-order cosine, (c) thirdorder sine, and (d) fifth-order sine wobble about a local $Z$ axis.

\subsection{Scale Errors in the Rotation Axis Encoder}

Description: Scale errors are errors in the angular encoder, i.e., errors in the index angle $\theta$; see Fig. 1(f). Angular scale errors result in a discrepancy between the intended and actual rotation angle of the stage. We consider individual harmonic components of the form $a \cos n \theta$ and $a \sin n \theta$, where $a$ is the amplitude, $n$ is the order of the harmonic, and $\theta$ is the index angle. The amplitude $a$ is $0.05^{\circ}$, and we consider low-order harmonics $n$ ranging from 1 to 10 .

Distance error: Figure 4 shows the lines with the highest sensitivity to second-order cosine and sine encoder scale errors; these are the face diagonals passing through the rotation axis in any given horizontal $(X Z)$ plane. The lines are oriented at $45^{\circ}$ and $135^{\circ}$ with respect to the $X$ axis for cosine scale error, whereas they are parallel and perpendicular to the plane of the detector for sine scale error (i.e., $0^{\circ}$ and $90^{\circ}$, respectively, with respect to the $X$ axis), when the reference object is located at an index angle of zero degrees. The magnitude of the distance error is $0.02 \mathrm{~mm}$ for the second harmonic, and the distance error 
sensitivity is therefore $0.4 \mathrm{~mm} /{ }^{\circ}$. The error is positive for one of the lines and negative for the other. The sign of the distance error reverses when the amplitude of the scale error is $-0.05^{\circ}$. First-order scale error results in a small distance error of $0.001 \mathrm{~mm}$, while higher harmonics produce negligible distance errors.

Form error: Despite the relatively large encoder scale error amplitude of $0.05^{\circ}$ considered in this paper, the magnitude of the resulting form error is lower than $0.05 \mathrm{~mm}$ for all harmonics. For any given order, the spheres farther away from the axis of rotation show larger form error than those closer to the axis.

\subsection{Sensitivity Matrix for Rotation Axis Errors}

We discussed the sensitivity of distance and form error to different rotation stage geometry errors in the previous subsections. In Table 1, we summarize these sensitivities in the form of a sensitivity matrix using the 16 spheres $(A-H, J-M, Q-T)$ shown in Fig. 8. Spheres $A, B, C$, and $D$ are located on the bottom plane $(Y=-25 \mathrm{~mm})$ and on a circle of $50 \mathrm{~mm}$ diameter. Spheres $E, F, G$, and $H$ are located on the top plane $(Y=25 \mathrm{~mm})$ and on a circle of $50 \mathrm{~mm}$ diameter. The spheres create four body diagonals $(A G, C E, D F, B H)$ and four face diagonals $(A C, B D, F H, E G)$.

Table 1. Sensitivity matrix for rotation axis errors based on the spheres shown in Fig. 8.

\begin{tabular}{|c|c|c|c|c|c|}
\hline \multirow[t]{2}{*}{ Source } & \multicolumn{2}{|c|}{ Distance error } & \multicolumn{2}{|c|}{ Form error } & \multirow[t]{2}{*}{ Description } \\
\hline & Lines & Sensitivity $^{\mathrm{a}}$ & Spheres $^{\mathrm{b}}$ & Sensitivity ${ }^{\mathrm{a}}$ & \\
\hline$Z$ location & $\begin{array}{l}A G, C E, \\
D F, B H\end{array}$ & $\begin{array}{c}0.17 \\
\mathrm{~mm} / \mathrm{mm}\end{array}$ & None & 0 & Distance error: Body diagonals through $P$ \\
\hline Axial & $\begin{array}{l}A G, C E \\
D F, B H\end{array}$ & $\begin{array}{c}0.1 \\
\mathrm{~mm} / \mathrm{mm}\end{array}$ & All & $\begin{array}{l}1 \mathrm{~mm} / \mathrm{mm} \\
\text { to } \\
2 \mathrm{~mm} / \mathrm{mm}\end{array}$ & $\begin{array}{l}\text { Distance error: First order, body diagonals through } P \\
\text { Form error: Depends on order, with higher sensitivity to } \\
\text { second, fourth, sixth, eighth order }\end{array}$ \\
\hline $\begin{array}{l}\text { Radial } \\
\text { component } \\
\text { along } X\end{array}$ & $\begin{array}{l}A C, B D \\
F H, E G \\
J L, K M \\
Q S, R T\end{array}$ & $\begin{array}{c}0.1 \\
\mathrm{~mm} / \mathrm{mm}\end{array}$ & All & $\begin{array}{l}1 \mathrm{~mm} / \mathrm{mm} \\
\text { to } \\
2 \mathrm{~mm} / \mathrm{mm}\end{array}$ & $\begin{array}{l}\text { Distance error: Second order, face diagonals through } P \\
\text { Form error: Depends on order, with higher sensitivity to } \\
\text { third, fifth, seventh order }\end{array}$ \\
\hline $\begin{array}{l}\text { Radial } \\
\text { component } \\
\text { along } Z\end{array}$ & $\begin{array}{l}A C, B D \\
F H, E G \\
J L, K M \\
Q S, R T\end{array}$ & $\begin{array}{c}0.1 \\
\mathrm{~mm} / \mathrm{mm}\end{array}$ & None & 0 & Distance error: Second order, face diagonals through $P$ \\
\hline $\begin{array}{l}\text { Wobble } \\
\text { about } X\end{array}$ & $\begin{array}{l}A G, C E, \\
D F, B H\end{array}$ & $0.3 \mathrm{~mm} /{ }^{\circ}$ & All & $\begin{array}{l}0.5 \mathrm{~mm} /{ }^{\circ} \\
\text { to } \\
0.75 \mathrm{~mm} /{ }^{\circ}\end{array}$ & $\begin{array}{l}\text { Distance error: First order, body diagonals through } P \\
\text { Form error: Depends on order, with higher sensitivity to } \\
\text { third, fifth, seventh order }\end{array}$ \\
\hline $\begin{array}{l}\text { Wobble } \\
\text { about } Z\end{array}$ & $\begin{array}{l}A G, C E \\
D F, B H\end{array}$ & $0.3 \mathrm{~mm} /{ }^{\circ}$ & All & $\begin{array}{l}0.5 \mathrm{~mm} /{ }^{\circ} \\
\text { to } \\
1.2 \mathrm{~mm} /{ }^{\circ}\end{array}$ & $\begin{array}{l}\text { Distance error: First order, body diagonals through } P \\
\text { Form error: Depends on order, with higher sensitivity to } \\
\text { third, fifth, seventh, ninth order }\end{array}$ \\
\hline Scale errors & $\begin{array}{l}A C, B D \\
F H, E G, \\
J L, K M \\
Q S, R T\end{array}$ & $0.4 \mathrm{~mm} /{ }^{\circ}$ & All & $1 \mathrm{~mm} /{ }^{\circ}$ & Distance error: Second order, face diagonals through $P$ \\
\hline
\end{tabular}

${ }^{a}$ Absolute magnitude is shown, i.e., without sign of sensitivity coefficient.

b 'All' in this column refers to the eight spheres in Fig. 8(a) and eight spheres in Fig. 8(b) only.

At the initial angular position of the object (index angle of $0^{\circ}$ ), spheres $A, C, E$, and $G$ form a plane parallel to the detector, while spheres $B, D, F$, and $H$ form a plane perpendicular to the detector.

Dimensional measurements of these spheres are most sensitive to the different detector geometry errors discussed in Part I [1]. These spheres are also sensitive to several rotation stage geometry errors. Figure 8(b) shows an additional eight spheres forming four face diagonals that are sensitive to rotation stage errors. Spheres $J, K, L$, and $M$ are located on the bottom $(Y=-25 \mathrm{~mm})$ plane and form a circle of $50 \mathrm{~mm}$ diameter. Spheres $Q, R, S$, and $T$ are on the top $(Y=25 \mathrm{~mm})$ plane and form a circle of $50 \mathrm{~mm}$ diameter. Dimensional measurements of these spheres are most sensitive to the remaining rotation stage errors. 
Here, we summarize the sensitivity observations made in the previous sections as they pertain to the sensitive spheres in the reference object. The table shows the magnitude of distance and form errors, along with the sensitivity coefficients, which are the ratio of the magnitude of the distance/form errors to the magnitude of the corresponding geometry error. The table shows that distance errors are sensitive to rotation stage $Z$ location error and some harmonics of axial, radial, wobble, and scale errors. Sphere form errors are relatively sensitive to certain orders of axial, radial, wobble, and scale errors.

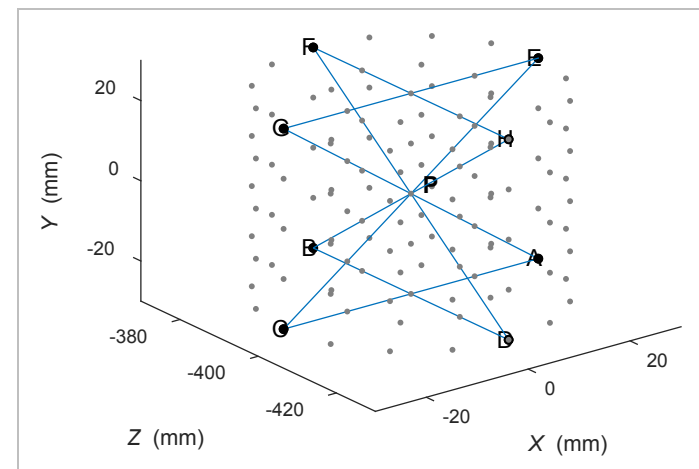

(a)

Fig. 8. (a) Eight of the 125 spheres form four face diagonals and four body diagonals that can be used to capture all detector geometry errors as shown in Part I [1] and some rotation stage errors. (b) Eight additional spheres forming four face diagonals can be used to detect the remaining rotation stage errors.

\section{Discussion}

\subsection{Sensitive Sphere Locations for Detector and Rotation Stage Errors}

Our recommendations and rationale for sphere center locations to detect detector and rotation stage errors are as follows (see Fig. 8):

- eight spheres located at index angles of $0^{\circ}, 45^{\circ}, 90^{\circ}, 135^{\circ}, 180^{\circ}, 225^{\circ}, 270^{\circ}$, and $315^{\circ}$, radially as far away from the axis of rotation as possible and in the lowest $Y$ position possible;

- eight spheres located at index angles of $0^{\circ}, 45^{\circ}, 90^{\circ}, 135^{\circ}, 180^{\circ}, 225^{\circ}, 270^{\circ}$, and $315^{\circ}$, radially as far away from the axis of rotation as possible and in the highest $Y$ position possible;

- eight spheres in the lower horizontal plane and eight spheres in the upper horizontal plane allowing for the determination of four face diagonals each ( $A C, B D, E G, F H, J L, K M, Q S, R T)$; and

- four spheres in the lower horizontal plane and four spheres in the upper horizontal plane in Fig. 8 (a) allowing for the determination of four body diagonals $(A G, C E, D F, B H)$.

- The face diagonals in the lowest and highest $Y$ positions and the body diagonals are recommended test positions to capture both detector and rotation stage errors in XCT instruments.

- The form error on the 16 spheres is sensitive to the different geometry errors discussed in this paper. Monitoring form error of spheres located in the measurement volume, especially those located further away from $P$ along both the radial and $Y$ directions, is therefore a quick and easy test of detector and rotation stage errors.

\subsection{Sensitivity Values at Other Rotation Axis and Detector Locations}

In Sec. 2, we reported the sensitivity values for the specific case of source to rotation axis and source to detector distances of $400 \mathrm{~mm}$ and $1177 \mathrm{~mm}$, respectively. While the simulations can easily be performed for other rotation axis and detector distances, for some geometry error parameters, the sensitivity values at 
other distances are either directly or inversely proportional to the magnification. This allows for efficient calculation of sensitivity values for any rotation axis and detector distances if sensitivity values are known for one combination of rotation axis and detector distances.

\subsection{Reference Object Design for Geometry Parameter Determination}

The idea of projecting sphere centers instead of the entire sphere provides for an efficient method to investigate suitable geometry for a reference object that is sensitive to instrument geometry errors. Ferrucci et al. [4] presented the design of one reference object and a method to determine the instrument geometry parameters. Their method relies on the acquisition of radiographs in the presence of noise, followed by determination of sphere centers and geometry parameter estimation by fitting to a model. Subsequently, they estimated the uncertainty in the parameters for 10 different cases where different systematic errors were introduced for each case.

Our method of projecting only sphere centers allows for the determination of instrument parameters in the presence of both systematic errors and noise without the need to generate radiographs, thus making it possible to perform a Monte Carlo simulation for thousands of conditions and for different reference object designs. Thus, it is possible to identify candidate reference object designs that yield the geometry parameters with the largest possible sensitivity.

\subsection{Validation of the Single-Point Ray Tracing Method}

We presented some examples validating the single-point ray tracing method in Part I. Here, we present additional examples that involve rotation stage errors.

In the presence of the first harmonic cosine term of the wobble error component along the $X$ axis of amplitude $0.2^{\circ}$, the magnitude of the error in the body diagonals shown in Fig. 2(a) is $0.062 \mathrm{~mm}$ using the radiograph-based method. The corresponding value obtained using the single-point ray tracing method is $0.061 \mathrm{~mm}$, which is in excellent agreement with the radiograph-based method.

Figure 9(a) shows the form error for one of the spheres in the presence of the third harmonic sine term of the radial error component along the $X$ axis using the radiograph-based method, while Fig. 9(b) shows the form error for the same sphere using the single-point ray tracing method. The form error is $0.2 \mathrm{~mm}$ for both methods.

Figure 9(c) shows the form error for one of the spheres in the presence of the second harmonic cosine term of the axial error using the radiograph-based method, while Fig. 9(d) shows the form error for the same sphere using the single-point ray tracing method. The form error is $0.16 \mathrm{~mm}$ for the radiograph-based method and $0.19 \mathrm{~mm}$ for the single-point ray tracing method, and so the agreement in the form error is within a few tens of micrometers.

\subsection{Experimental Validation}

In this study, we used our single-point ray tracing method to identify optimal sphere center locations that are sensitive to XCT instrument geometry errors. No radiographs are required for this method, and so no reconstruction is needed. We verified our method for all of the geometry errors presented in this paper through the radiograph-based method (see previous subsection for some examples), where radiographs are generated numerically and reconstructed using commercially available software. 


\section{Journal of Research of the National Institute of Standards and Technology}

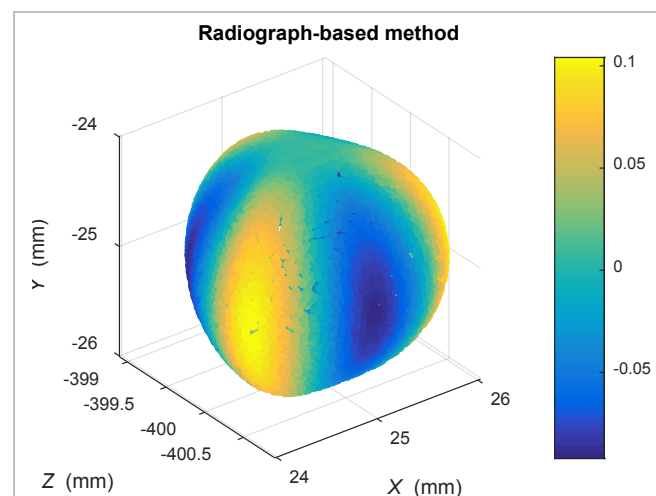

(a)

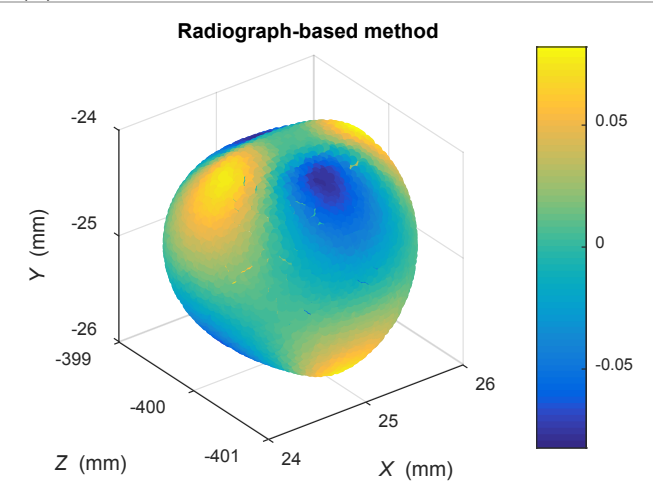

(c)

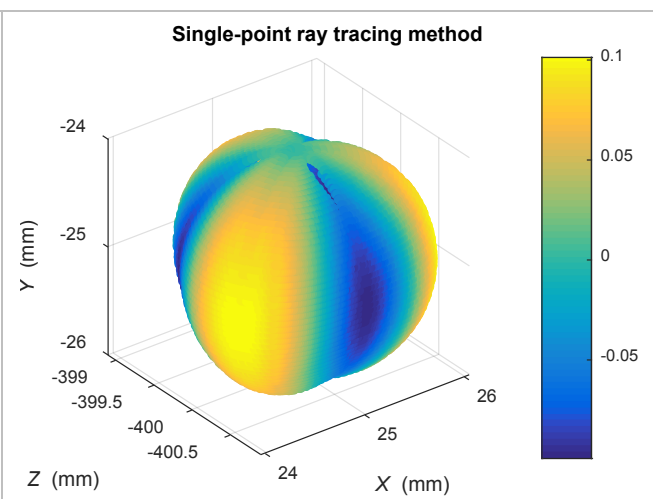

(b)

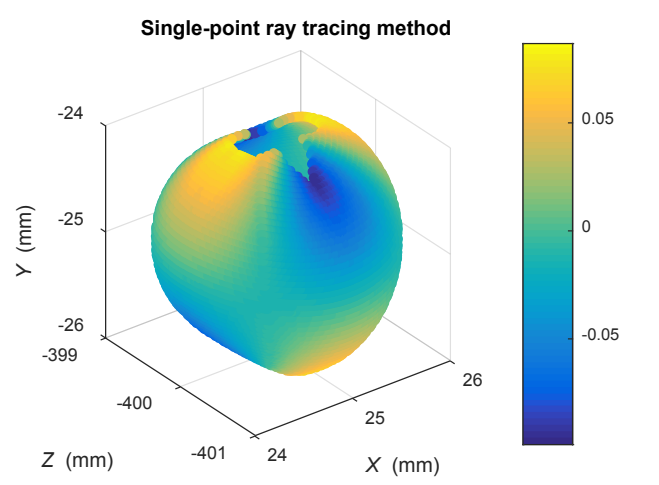

(d)

Fig. 9. (a) Form error for a sphere in the presence of the third harmonic sine term of the radial error component along the $X$ axis using the radiograph-based method. (b) Form error for the same sphere using the single-point ray tracing method. (e) Form error for a sphere in the presence of the second harmonic cosine term of the axial error using the radiograph-based method. (d) Form error for the same sphere using the single-point ray tracing method.

It is possible to test some of the claims made in this paper experimentally. For example, radiographs can be acquired at certain rotation axis and detector distances, but the reconstruction software can be provided incorrect values for these distances, thus confirming the sensitivity claims made here. We are in the process of performing such experiments and have reported preliminary results in Ref. [5]. We have also used third-party software, e.g., aRTist, ${ }^{2}$ to generate radiographs for some cases, and the results agree with both the radiograph-based method and our single-point ray tracing method.

\section{Conclusions}

In Part II of this study, we have described the effect of rotation stage errors in XCT instruments on sphere center-to-center distance errors and sphere form errors for spheres located in the measurement volume for the specific case of source to rotation axis and detector distances of $400 \mathrm{~mm}$ and $1177 \mathrm{~mm}$, respectively. In particular, we considered errors associated with the rotation axis such as its location error, and axial, radial, wobble, and scale errors. We performed our simulations using two different approaches, the radiograph-based method and the single-point ray tracing method.

\footnotetext{
${ }^{2}$ Certain commercial equipment, instruments, or materials are identified in this paper to foster understanding. Such identification does not imply recommendation or endorsement by the National Institute of Standards and Technology, nor does it imply that the materials or equipment identified are necessarily the best available for the purpose.
} 
The radiograph-based method involves generating radiographs of the spheres in the presence of geometry errors, performing reconstruction from the radiographs assuming ideal geometry, and performing surface determination and fitting to determine sphere centers, followed by calculation of sphere center-tocenter distance error and sphere form error. This method is labor-intensive and takes several hours on a personal computer for each set of radiographs.

In Part I, we therefore reported on a novel single-point ray tracing method where sphere centers are forward projected onto the detector in the presence of geometry errors. We then back project the rays assuming ideal geometry to determine sphere center locations using a least-squares approach. This method does not involve any reconstruction and therefore only takes a few minutes on a personal computer.

Using the single-point ray tracing method (and validated using the radiograph-based method), we quantified the sensitivity of the distance and form error to each of the rotation stage error parameters considered in this paper. Based on the analysis, we made recommendations on the optimal positions of spheres in the measurement volume to detect these geometry errors. The motivation for this work is to provide input to ongoing standards efforts within ASME and ISO so that the test procedures they specify are sensitive to all known geometry errors.

\section{Acknowledgments}

The first author is grateful to Massimiliano Ferrucci of KU Leuven for helpful discussions during the course of this work and for his careful review of the paper. The authors thank Dr. Craig Shakarji of the National Institute of Standards and Technology (NIST), Dr. Valentina Aloisi of North Star Imaging, Inc., Dr. Toshiyuki Takatsuji of the National Metrology Institute of Japan (NMIJ), and Dr. Herminso VillarragaGómez of Nikon Metrology, Inc., for their feedback.

\section{References}

[1] Muralikrishnan B, Shilling M, Phillips S, Ren W, Lee V, Kim F (2019) X-ray computed tomography instrument performance evaluation, Part I: Sensitivity to detector geometry errors. Journal of Research of the National Institute of Standards and Technology 124:124014. https://doi.org/10.6028/jres.124.014

[2] Marsh E (2008) Precision Spindle Metrology (DEStech Publications, Inc., Lancaster, PA).

[3] Ferrucci M, Ametova E, Probst G, Craeghs T, Dewulf W (2018) Sensitivity of CT dimensional measurements to rotation stage errors. Proceedings of the 8th Conference on Industrial Computed Tomography (Wels, Austria).

[4] Ferrucci M, Heřmánek P, Ametova E, Carmignato S, Dewulf W (2018) Measurement of the X-ray computed tomography instrument geometry by minimization of reprojection errors-Implementation on simulated data. Precision Engineering 54:720. https://doi.org/10.1016/j.precisioneng.2018.03.012

[5] Muralikrishnan B, Shilling M, Phillips S, Ren W, Lee V, Kim F, Alberts G, Aloisi V (2019) X-ray computed tomography instrument performance evaluation: Detecting geometry errors using a calibrated artifact. SPIE Conference on Defense and Commercial Sensing (Baltimore, MD). https://doi.org/10.1117/12.2518108

\footnotetext{
About the authors: Bala Muralikrishnan and Meghan Shilling are mechanical engineers in the Sensor Science Division at NIST. Steve Phillips was a physicist in the Sensor Science Division at NIST. Wei Ren is a computer scientist in the Sensor Science Division at NIST. Vincent Lee is a mechanical engineer in the Sensor Science Division at NIST. Felix Kim is a mechanical engineer in the Intelligent Systems Division at NIST. The National Institute of Standards and Technology is an agency of the U.S. Department of Commerce.
} 